\title{
Snapshots of cooperative atomic motions in the optical suppression of charge density waves
}

\author{
Maximilian Eichberger ${ }^{1}$, Hanjo Schäfer ${ }^{1 *}$, Marina Krumova ${ }^{2}$, Markus Beyer $^{1}$, Jure Demsar ${ }^{1,3}$, Helmuth Berger ${ }^{4}$, \\ Gustavo Moriena ${ }^{5,6}$, Germán Sciaini ${ }^{5,6 *}$ \& R. J. Dwayne Miller ${ }^{5,6}$
}

\begin{abstract}
Macroscopic quantum phenomena such as high temperature superconductivity, colossal magnetoresistance, ferrimagnetism and ferromagnetism arise from a delicate balance of different interactions among electrons, phonons and spins on the nano scale $^{1}$. The study of the interplay among these various degrees of freedom in strongly coupled electron lattice systems is thus crucial to their understanding and for optimizing their properties. Charge density wave (CDW) materials ${ }^{2}$, with their inherent modulation of the electron density and associated periodic lattice distortion, represent ideal model systems for the study of such highly cooperative phenomena. With femtosecond time resolved techniques, it is possible to observe these interactions directly by abruptly perturbing the electronic distribution while keeping track of energy relaxation pathways and coupling strengths among the different subsystems ${ }^{3}{ }^{7}$. Numerous time resolved experiments have been performed on $\mathrm{CDWs}^{8}{ }^{13}$, probing the dynamics of the electronic subsystem. However, the dynamics of the periodic lattice distortion have been only indirectly inferred ${ }^{14}$. Here we provide direct atomic level information on the structural dynamics by using femtosecond electron diffraction ${ }^{15}$ to study the quasi two dimensional CDW system $1 \mathrm{~T} \mathrm{TaS}_{2}$. Effectively, we have directly observed the atomic motions that result from the optically induced change in the electronic spatial distribution. The periodic lattice distortion, which has an amplitude of $\sim 0.1 \AA$, is suppressed by about $20 \%$ on a timescale ( $\sim 250$ femtoseconds) comparable to half the period of the corresponding collective mode. These highly cooperative, electronically driven atomic motions are accompan ied by a rapid electron phonon energy transfer ( $\sim 350$ femtose conds) and are followed by fast recovery of the CDW ( $\sim 4$ picoseconds). The degree of cooperativity in the observed struc tural dynamics is remarkable and illustrates the importance of obtaining atomic level perspectives of the processes directing the physics of strongly correlated systems.
\end{abstract}

IT $\mathrm{TaS}_{2}$ is one of the most studied quasi two dimensional CDW systems ${ }^{16}{ }^{19}$. It has a simple crystalline structure, consisting of planes of hexagonally arranged tantalum (Ta) atoms, sandwiched by two sul phur (S) layers coordinating the central Ta atom in an octahedral arrangement ${ }^{16,17}$. In the low temperature CDW phase, the conduction electron density becomes modulated, modifying the forces among the ions and generating a periodic lattice distortion (PLD) with a peri odicity of $\sim 12 \AA$. This effect is illustrated in Fig. 1a, b together with the corresponding potential energy surfaces, $U(Q)$, where $Q$ is the generalized coordinate of the atomic displacements. The correspond ing changes in $U(Q)$ result in a shift of the equilibrium atomic positions to introduce a PLD. In $1 \mathrm{~T} \mathrm{TaS}_{2}$, the transition from its metallic, unmodulated, phase to an incommensurate CDW phase (ICP) happens at $550 \mathrm{~K}$. At $350 \mathrm{~K}$, a transition to a nearly commensurate CDW phase (NCCP) occurs, where the amplitude of the PLD increases abruptly from 0.03 to $0.1 \AA$ and the CDW wavevector undergoes a sudden angular rotation from $\phi \quad 0^{\circ}$ to $\sim 123^{\circ}$ with respect to the fun damental lattice vector of the host (unreconstructed) lattice. Finally, a transition to a commensurate CDW phase (CCP) takes place at $180 \mathrm{~K}$, with $\phi \quad 13.9^{\circ}$ and a $\sqrt{ } 13 \times \sqrt{ } 13$ periodicity ${ }^{17}$. This phase transition is characterized by the appearance of the gap throughout the Fermi surface, and is argued to be due to Mott localization ${ }^{20}$. The appearance of the ICP can be described by the standard Peierls model ${ }^{2}$. According to this, in low dimensional systems the divergence in the static electronic susceptibility at the wavevector $2 \mathbf{k}_{\mathrm{F}}$, connecting par allel Fermi surfaces at $\mathbf{k}_{\mathrm{F}}$ and $\mathbf{k}_{\mathrm{F}}$ (where $\mathbf{k}_{\mathrm{F}}$ is the Fermi wavevector), gives rise to an instability of conduction electrons against the formation of the electron density modulation. Indeed, the comparison of the topology of the Fermi surface with parallel sections that can be con nected by the modulation wavevector favours the standard Peierls model for the emergence of the ICP ${ }^{16}{ }^{18}$. The nature of the CCP and
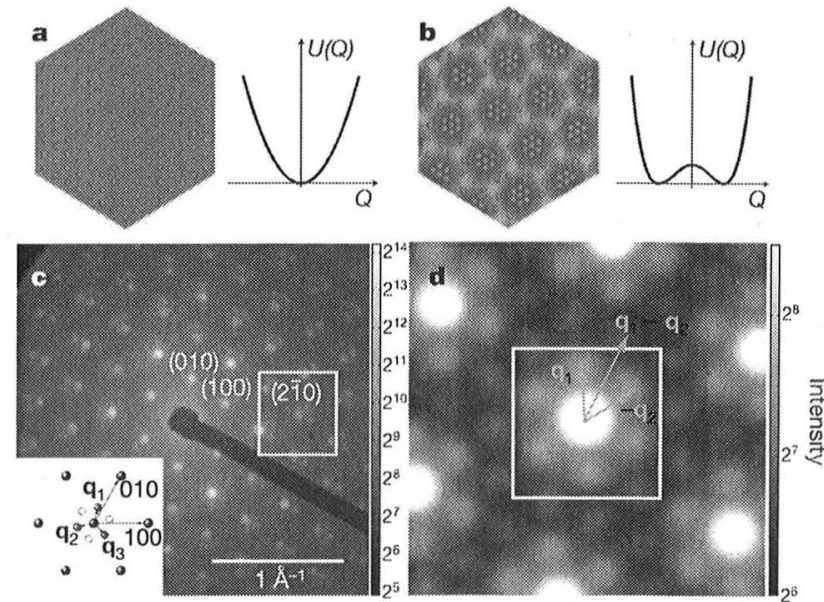

Figure 1 | FED data in the NCCP in 1 T $\mathrm{TaS}_{2}$. a, b, Schematic real space images of Ta atoms and the electron density in the metallic (a) and the CDW (b) states together with the corresponding potential energy, $U$, as a function of generalized coordinate, Q. c, The diffraction pattern of $1 \mathrm{~T} \mathrm{TaS}_{2}$ at $200 \mathrm{~K}$ (intensity is shown on a logarithmic scale in arbitrary units). Each Bragg reflection is surrounded by six first order CDW reflections at the scattering wave vectors $q_{i}$, which each has an out of plane component of $\pm 1 / 3 c^{*}$ (red and blue circles, respectively, in inset). The projections of the $\mathbf{q}_{i}$ on the basal plane, with a modulus of $\sim 0.28 a^{*}$, are tilted away from the closest fundamental lattice vector by an angle $\phi \approx 12.3^{\circ}$. $\mathrm{d}$, Magnified view of the diffraction intensity $(I)$ near the (210) Bragg peak (see box in c; for presentation purposes the diffraction image was symmetrized with respect to the six fold axis). The secondary CDW reflections at the wavevector corresponding to the difference of the wavevectors of the first order CDW peaks ${ }^{20}$ are clearly resolved.

${ }^{i}$ Physics Department and Center of Applied Photonics and Zukunftskolleg, University of Konstanz, D-78457 Konstanz, Germany. ${ }^{2}$ Department of Chemistry, University of Konstanz, D-78457 Konstanz. Germany. ${ }^{3}$ Complex Matter Department, Jozef Stefan Institute, SI-1000 Ljubljana, Slovenia. ${ }^{4}$ Physics Department, EPFL CH-1015 Lausanne, Switzerland. ${ }^{5}$ Institute for Optical Sciences and Departments of Chemistry and Physics, University of Toronto. Toronto, Ontario M5S 3H6. Canada. ${ }^{6}$ Max Planck Research Department for Structural Dynamics, Department of Physics, University of Hamburg, Centre for Free Electron Laser Science, DESY, D-22607 Hamburg, Germany.

"These authors contributed equally to this work 
the NCCP, as well as of the ICP NCCP and NCCP CCP transitions, is, however, still under debate ${ }^{18}$. Recently, $1 \mathrm{~T} \mathrm{TaS}_{2}$ received additional attention owing to the observation there of superconductivity below $5 \mathrm{~K}$ under high pressure ${ }^{19}$.

In this study, we investigated the structural dynamics of the PLD in $30 \mathrm{~nm}$ thick, free standing slices of $1 \mathrm{~T} \mathrm{TaS}$. We performed femto second electron diffraction (FED) experiments in transmission geo metry along the $c$ axis, that is, perpendicular to the $\mathrm{TaS}_{2}$ layers. The films were photoexcited with $140 \mathrm{fs}$ optical pulses, and $50 \mathrm{keV}$ elec trons, in bursts of $\leq 250 \mathrm{fs}$, were used to monitor the structural changes by recording time delayed diffraction patterns. The diffraction pattern of the NCCP $(200 \mathrm{~K})$ recorded in this set up is shown in Fig. 1c together with the assignment of some of the scattering vectors. The intense peaks are the Bragg reflections of the host lattice. Each of the Bragg peaks is surrounded by six weak satellite peaks originating from the PLD, with modulation wavevectors $\mathbf{q}_{i}$ (ref. 21$)$, illustrated in Fig. 1c (inset) and Fig. 1d.

Figure $2 \mathrm{a}$ e shows the time evolution of the relative change of the diffraction signal in the vicinity of a Bragg peak, following photoexci tation. The corresponding traces of the relative changes in the Bragg peaks $\left(\Delta I_{\text {Bragg }} / I_{\text {Bragg }}\right)$, the inelastic background $\left(\Delta I_{\text {bckg }} / I_{\text {bckg }}\right)$ and the CDW peaks $\left(\Delta I_{\mathrm{CDW}} / I_{\mathrm{CDW}}\right)$ are shown in Fig. $2 \mathrm{f}$ (see also Sup plementary Fig. 3 ). The intensity of the CDW peaks (the satellites of the Bragg peaks), $I_{\mathrm{CDW}}$, is suppressed by $\sim 30 \%$ on the timescale of hundreds of femtoseconds. The corresponding suppression of the PLD gives rise to more efficient scattering into the Bragg reflections of the host lattice, manifested by an increase of the Bragg peak intensity, $I_{\text {Bragg, }}$ by $\sim 15 \%$. In the CDW state, the presence of the PLD suppresses $I_{\mathrm{Bragg}}$ similarly to the effect of thermally induced disorder; that is, the presence of PLD can be looked upon as an effective Debye Waller effect. The decrease in $I_{\mathrm{CDW}}$ and the accompanying increase in $I_{\mathrm{Bragg}}$ thus illustrate a cooperative phenomenon in which the optically induced redistribution of electron density efficiently decreases the PLD amplitude. Because $I_{\mathrm{CDW}}$ is proportional to the square of the atomic displacements ${ }^{22}$, the resulting suppression of $I_{\mathrm{CDW}}$, by $\sim 30 \%$, corresponds to $\sim 16 \%$ change in atomic displacements $(\sim 0.02 \AA)$. Following the initial increase, $I_{\mathrm{Bragg}}$ is found to partially recover on the $350 \mathrm{fs}$ timescale. This recovery is accompanied by an increase in the inelastic background intensity, $I_{\mathrm{bckg}}$ see the intensity changes in the area indicated by the circle in Fig. $2 \mathrm{e}$ for the frames between 300 (Fig. 2c) and 5,800 fs (Fig. 2e). This process can be attributed to the generation of phonons with non zero momentum $(q \neq 0)$; hence, $I_{\text {Bragg }}$ is reduced owing to the conventional Debye Waller effect, leading to an increase in the inelastic background.

A noteworthy feature of the data shown in Fig. 2f, and elaborated on in Fig. 3a, is the apparent difference between the dynamics of $\Delta I_{\mathrm{Bragg}}$ $I_{\mathrm{Bragg}}$ and $\Delta I_{\mathrm{CDW}} / I_{\mathrm{CDW}}$. Although the maximum in $I_{\mathrm{Bragg}}$ is reached at a time delay of $\sim 300 \mathrm{fs}$ (Fig. 3a, dashed vertical line), the minimum in $I_{\mathrm{CDW}}$ is reached at a time delay of $\sim 500 \mathrm{fs}$ (Fig. 3a, solid vertical line), at which point $I_{\mathrm{Bragg}}$ has decreased from its maximum. This difference can be naturally explained by considering the effects of both the sup pression of the PLD and the increase in the $q \neq 0$ phonon population on the two diffraction intensities. For the case of $I_{\mathrm{Bragg}}$, the first effect gives rise to its increase as the periodicity of the host lattice is enhanced, and the increase in the $q \neq 0$ phonon population (the Debye Waller effect) has the opposite effect. Indeed, from the fast recovery of $I_{\text {Bragy }}$ and the corresponding increase in $I_{\mathrm{bckg}}$ it follows that the energy transfer from electrons to $q \neq 0$ phonons in $1 \mathrm{~T} \mathrm{TaS}_{2}$ takes place on the timescale of a few hundred femtoseconds $\left(\tau_{\mathrm{c}-\mathrm{ph}} \approx 350 \mathrm{fs}\right)$. For $I_{\mathrm{CDW}}$, both the displacive excitation of highly correlated atomic motions and phonon induced disorder contribute to its suppression, explaining the longer timescale on which the minimum of $I_{\mathrm{CDW}}$ is reached.

It is instructive to compare the structural dynamics data with those of the electronic subsystem. We have performed all optical pump probe measurements, where the dynamics are mainly sensitive to the changes in the electronic properties. The photoinduced reflectivity change (Fig. $2 \mathrm{~g}$ ) shows a rapid onset on the $100 \mathrm{fs}$ timescale, followed by a fast recovery with a decay time of $150 \mathrm{fs}$ and subsequent slower decay with a relaxation time of $\sim 4 \mathrm{ps}$, which is nearly identical to the
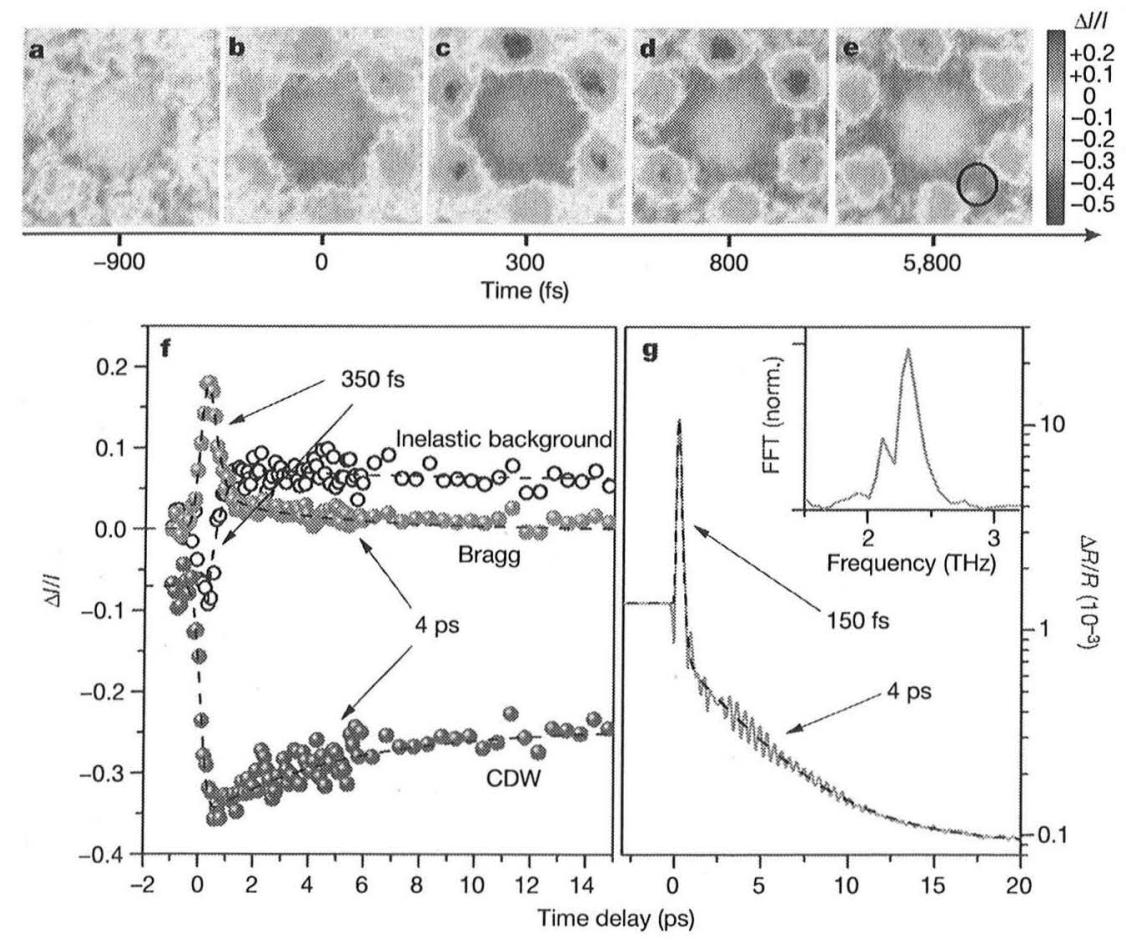

2 0.1 .1 (1)

(1)
photoexcitation with a fluence of $2.4 \mathrm{~mJ} \mathrm{~cm}{ }^{2}$ and a Fig 4 energy of $3.2 \mathrm{eV}$ (see also Supplementary images were obtained by averaging (area enclosed by the box in Fig. 1d) over all individual Bragg reflections to increase the signal to noise ratio. The circle in e represents the area over which the inelastic background intensity was monitored.

f, Corresponding dynamics of $\Delta I_{\mathrm{Bragg}} / I_{\mathrm{Bragg}}, \Delta I_{\mathrm{CDW}} /$ $I_{\mathrm{CDW}}$ and $\Delta I_{\mathrm{bckg}} / I_{\mathrm{bckg}}$ with fits to the data (dashed lines) and the extracted timescales. The suppression of the PLD, that is, the CDW peak intensity at negative time delays, is due to an increase in the sample temperature caused by the photoexcitation pulse train (accumulative heating). The initial drop in $I_{\text {bckg }}$ is an artefact, a result of the decrease in the diffraction intensity of the nearby CDW peaks, whose tails extend well into the region where the inelastic background was evaluated (e). g, Dynamics of the differential reflectivity change, $\Delta R / R$, at $1.55 \mathrm{eV}(800 \mathrm{~nm})$, recorded at the same initial temperature and the same excitation energy density, together with the fit (dashed line). The signal has been offset vertically for presentation purposes. The oscillatory response corresponds to the coherently excited amplitude mode at $2.3 \mathrm{THz}$ and phonon mode at $2.1 \mathrm{THz}$ (refs 9, 11,21). Inset, fast Fourier transform (FFT) of the oscillatory component. 

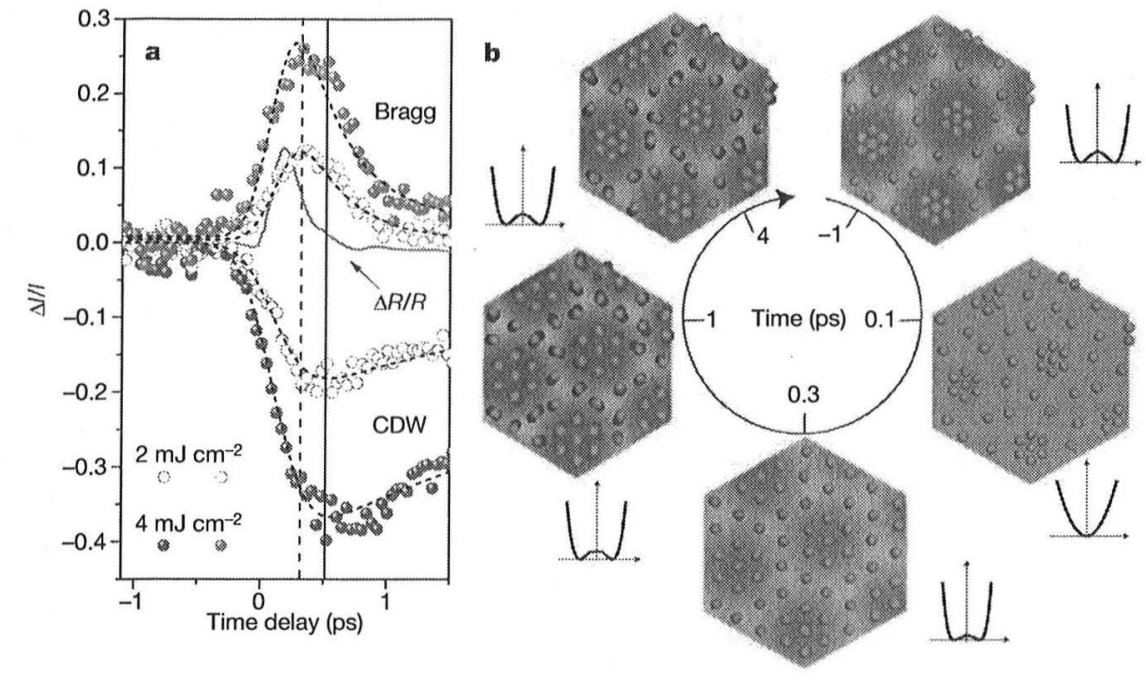

Figure 3 | Early time dynamics and emerging time evolution of the CDW state in $1 \mathrm{~T} \mathrm{TaS}_{2}$ on photoexcitation. a, Data were recorded with a $40 \mathrm{fs}$ time step at two excitation fluences, and compared with the optical $\triangle R / R$ data. The maximum induced changes in the Bragg (dashed vertical line) and CDW (solid vertical line) peaks were achieved $\sim 300 \mathrm{fs}$ and, respectively, $\sim 500 \mathrm{fs}$ after photoexcitation. $\mathbf{b}$, The evolution of the real space structure of the Ta plane of $1 \mathrm{~T} \mathrm{TaS}_{2}$ following photoexcitation with an intense optical pulse (circles represent Ta atoms and blue shading represents the density of conduction electrons; the amplitudes are strongly exaggerated). Before photoexcitation $(t \approx-1 \mathrm{ps})$, the Ta atoms are periodically displaced from their pure 1T structure, forming a nearly commensurate $\mathrm{CDW}$. Intense perturbation of the electronic system gives rise to smearing of the electron density modulation $(t \approx 0.1 \mathrm{ps})$, driving the lattice towards the undistorted state (at $t \approx 0.3 \mathrm{ps}$, the hexagonal symmetry of the pure 1T phase is nearly recovered). In parallel, the energy is transferred from the electronic subsystem to phonons on the $300 \mathrm{fs}$ timescale, resulting in recovery of the electron density modulation and thermal disordering of the lattice ( $t \approx 1 \mathrm{ps}$ ). The CDW order is recovered at $t \approx 4 \mathrm{ps,}$ after which time the sample is thermalized at a somewhat higher temperature.
CDW recovery time observed in the FED studies. The short decay timescale is identical to the one obtained in the NCCP by time and angle resolved photoemission spectroscopy" (tr ARPES) and can be attributed to the electron phonon energy transfer. Because the electron phonon scattering rate is strongly momentum dependent $(\propto 1 / q)$, it is quite natural to observe shorter time constants in optics and tr ARPES than in FED. In the former experiments the signal is dominated by the energy transfer to $q \approx 0$ phonons, whereas in the latter the behaviour of $I_{\mathrm{Bragg}}$ and $I_{\mathrm{bckg}}$ is governed by the population of large $q$ phonons. In the optical data, owing to their high signal to noise ratio, in addition to the electronic response a weak oscillatory signal is observed. The main mode observed at $2.3 \mathrm{THz}$ is the totally symmetric amplitude mode $\mathrm{e}^{9,11,23}$ of the $\mathrm{CDW}$, whose amplitude is apparently smaller than the noise level in the FED data.

Despite the fact that a large amount of energy is transferred to phonons on the subpicosecond timescale, the system is not yet in thermal equilibrium 1 ps after photoexcitation. The recovery of the PLD amplitude is clearly observed in $I_{\mathrm{Bragg}}$ and $I_{\mathrm{CDW}}$. This timescale is well decoupled from both subpicosecond timescales. By fitting the recovery of $I_{\mathrm{CDW}}$ with an exponential decay, we obtain a $\mathrm{CDW}$ recovery time of $\tau_{\text {rec }} \approx 4 \mathrm{ps}$. As this timescale is much longer than the oscillation period of the amplitude mode, it is reasonable to assume that the electronic part of the order parameter follows the PLD on the aforementioned timescale. Here the process that governs the CDW recovery dynamics is the thermalization with the longer wavelength acoustic phonons by means of anharmonic phonon decay. Indeed, the characteristic linewidths of the low energy optical phonon $s^{23}$ are about $10 \mathrm{~cm}{ }^{1}$, corresponding to lifetimes of 3 ps. The two distinct relaxation timescales, one of the order of $100 \mathrm{fs}$ and the other of several picose conds, are commonly observed in optical experiments in CDWs ${ }^{8,10}$. From the direct structural dynamics and optical data on $1 \mathrm{~T} \mathrm{TaS}_{2}$, we can conclude that the longer timescale describes the recovery of the coupled electron lattice order parameter and that the shorter timescale corresponds to the partial recovery of the electronic part alone $e^{14}$.

To determine the time constant for the electronic suppression of the PLD, which leads to an increase in $I_{\mathrm{Bragg}}$, we analysed its dynamics. By fitting (Supplementary Fig. 5 and Supplementary Information) the $I_{\text {Bragg }}$ trace, taking into account the finite optical and electron pulse widths, we determined a timescale of $\tau_{\text {supp }} \approx 250 \pm 70 \mathrm{fs}$ for the PLD suppression.
Information complementary to the above findings comes from con sidering the energy flow following photoexcitation. In the experiments with fluences $F \quad 24 \mathrm{~mJ} \mathrm{~cm}^{2}$, no signature of the NCCP ICP transi tion is observed. Only at $F \quad 4.8 \mathrm{~mJ} \mathrm{~cm}{ }^{2}$ is the photoinduced NCCP ICP transition realized (Supplementary Fig. 6 and Supplementary Information), as demonstrated by a strong suppression of the CDW peak intensity and a rotation of the primary CDW wavevectors, $\mathbf{q}_{i}$, by $\phi \approx 10^{\circ}$. Using the literature values of the optical constants and the overall specific heat (Supplementary Information), we obtained a tem perature increase of $\sim 180 \mathrm{~K}$ at $F \quad 4.8 \mathrm{~mJ} \mathrm{~cm}{ }^{2}$. This implies that the energy needed to drive the phase transition is comparable to the energy required simply to heat the sample across the phase transition. The rapid energy transfer from the electronic system to phonons $\left(\tau_{\mathrm{e}-\mathrm{ph}} \approx 150350 \mathrm{fs}\right)$, which is competing with the electronically driven PLD suppression process $\left(\tau_{\text {supp }} \approx 250 \mathrm{fs}\right.$ ), and the fact that the elec tronically excited symmetric amplitude mode does not map into the rotation of the CDW wavevector, suggest that the NCCP ICP transi tion can be driven only thermally.

The direct structural information obtained with FED, supported by time resolved optical and published tr ARPES $^{11}$ data, enabled us to elucidate the dynamics of the coupled electron lattice order parameter (Fig. 3b). Strong photoexcitation and subsequent electron electron scattering creates a high density of electron hole pairs within $\leq 100 \mathrm{fs}$, raising the effective electronic temperature to several thou sand kelvin. The electronic modulation is thereby strongly sup pressed, modifying the potential energy surface $U(Q)$. The collapse of the double well potential brings about highly cooperative atomic motions towards a new quasi equilibrium. This coherent process is, however, accompanied by the rapid recovery of the double well potential due to cooling of the electronic subsystem through the electron phonon scattering, which also takes place on the subpico second timescale ${ }^{813}$. The resulting suppression of the PLD ampli tude, by $\sim 20 \%(0.02 \AA)$, happens within a time of $\tau_{\text {supp }} \approx 250 \pm 70 \mathrm{fs}$, that is, about half the period of the amplitude mode, $\sim 440 \mathrm{fs}$ (refs 9 , 11). After a time delay of $t \approx 300 \mathrm{fs}$, the periodicity of the underlying lattice has increased and the amplitude of the PLD has decreased. By $t \approx 1 \mathrm{ps}$, the electronic modulation has been largely recovered and the electrons have transferred the energy to $q \neq 0$ phonons, randomizing the atomic motions. Finally, the coupled electron lattice order para meter recovers on the timescale of $\sim 4 \mathrm{ps}$, when the excess energy is 
redistributed by further thermalization with low energy acoustic phonons.

These results demonstrate the extreme robustness of the PLD in 1T $\mathrm{TaS}_{2}$ against electronic excitation triggered by a femtosecond optical pulse. By contrast, in the insulating CCP the gap is fully suppressed ${ }^{11}$ at only one tenth of the absorbed energy density used in our study. The large difference in the two energy densities presents a strong argument that the NCCP CCP transition is indeed Mott driven ${ }^{11,19}$.

The present work illustrates the importance of directly observing atomic motions on timescales short enough to follow even the effect of non equilibrium electronic distributions on strongly correlated lattice dynamics. In this respect, the introduction of table top FED systems $\mathrm{s}^{24} 26$ with sufficient brightness and time resolution ${ }^{24,26}$ is opening new path ways to the investigation of a myriad of cooperative systems in which electron lattice correlations have an important role ${ }^{26}{ }^{30}$. In systems with reduced dimensionality, such as quasi one dimensional and quasi two dimensional systems, in which structural changes have a predominantly in plane character, the use of FED may be particularly advantageous. Because information about structural dynamics over the entire two dimensional Brillouin zone is obtained in a single experi mental run by FED, it is easy to distinguish between different processes that give rise to changes in the diffraction intensities, as in the case of $1 \mathrm{~T} \mathrm{TaS}_{2}$. Moreover, with further instrumental improvements, for example an increase in the signal to noise ratio, FED could be used to find signatures of lattice modulations, which may be difficult to determine by means of static diffraction methods, much like modu lation optical spectroscopy is used to determine the electronic band structure in solids.

\section{METHODS SUMMARY}

In the present study, we used electron bunches of $\leq 250 \mathrm{fs}$ duration containing 4,000 electrons, each of which had a kinetic energy of $50 \mathrm{keV}$. The electron beam (spot size, $150 \mu \mathrm{m}$ ) was collimated by a magnetic lens to scatter from the sample and generate a diffraction pattern downstream. The diffraction patterns formed on a phosphor screen after being intensified by a multichannel plate, and were recorded using a charge coupled device camera. The background pressures were $10^{9}$ and $10^{7}$ mbar in the electron gun and the sample chamber sections, respect ively. We made the measurements in transmission mode at a repetition rate of $1 \mathrm{kHz}$. In this geometrical configuration, spatiotemporal mismatch and surface charging effects are negligible. The electron pulse duration was characterized using a recently developed electron/laser pulse cross correlation method based on pon deromotive scattering and $N$ body simulations. Photoinduced structural changes were initiated by $387 \mathrm{~nm}, 140 \mathrm{fs}$ pump pulses focused to a spot with a full width at half maximum of $350 \mu \mathrm{m}$. The overall instrumental response time was $240290 \mathrm{fs}$. The sample temperature, of $200 \mathrm{~K}$, was achieved by using a cold finger attached to a well conducting sample holder made of oxygen free copper. We measured the temperature in situ using a calibrated temperature sensor. The $30 \mathrm{~nm}$ thick, sin gle crystalline $1 \mathrm{~T} \mathrm{TaS}$ slices, $\sim 200 \mu \mathrm{m} \times 200 \mu \mathrm{m}$ in size (Supplementary Figs and 3), were obtained by cleaving a thicker single crystal using an ultramicrotome The slices were picked up from the water surface using a host copper mesh. All optical measurements were performed in reflection geometry using $60 \mathrm{fs}$ optical pulses (carrier wavelength, $800 \mathrm{~nm}$ ) at a repetition rate of $100 \mathrm{kHz}$. FED and all optical experiments were carried out with the same excitation energy density and under the same sample temperature conditions.

Received 17 May; accepted 24 September 2010.

Published online 24 November 2010.

1. Imada, M., Fujimori, A. \& Tokura, Y. Metal insulator transitions. Rev. Mod. Phys. 70 $10391263(1998)$

2. Grüner, G. Density Waves in Solids (Addison Wesley, 1994).

3. Kusar, P. et al. Controlled vaporization of the superconducting condensate in cuprate superconductors by femtosecond photoexcitation. Phys. Rev. Lett. 101 227001 (2008).

4. Ogasawara, T. et al. General features of photoinduced spin dynamics in

ferromagnetic and ferrimagnetic compounds. Phys. Rev. Lett. 94, 087202 (2005).

5. Averitt, R. D. et al. Ultrafast conductivity dynamics in colossal magnetoresistance manganites. Phys. Rev. Lett. 87, 017401 (2001)
6. Rini, M. et al. Control of the electronic phase of a manganite by mode selective vibrational excitation. Nature 449, 7274 (2007)

7. Kübler, C. et al. Coherent structural dynamics and electronic correlations during an ultrafastinsulator to metal phasetransition in $\mathrm{VO}_{2}$. Phys. Rev. Lett.99, 116401(2007).

8. Demsar, J., Biljakovic, K. \& Mihailovic, D. Single particle and collective excitations in the one dimensional charge density wave solid $\mathrm{K}_{03} \mathrm{MoO}_{3}$ probed in real time by femtosecond spectroscopy. Phys. Rev. Lett. 83, 800803 (1999).

9. Demsar, J. et al. Femtosecond snapshots of gap forming charge density wave correlations in quasi two dimensional dichalcogenides $1 \mathrm{~T} \mathrm{TaS} 2$ and $2 \mathrm{H} \mathrm{TaSe}_{2}$. Phys. Rev. B 66, 041101 (2002).

10. Yusupov, R.V. et al. Single particle and collective mode couplings associated with 1 and 2 directional electronic ordering in metallic $\mathrm{RTe}_{3}(\mathrm{R}=\mathrm{Ho}$, Dy, Tb). Phys. Rev. Lett. 101, 246402 (2008).

11. Perfetti, L. et al. Time evolution of the electronic structure of $1 \mathrm{~T} \mathrm{TaS}_{2}$ through the insulator metal transition. Phys. Rev. Lett. 97, 067402 (2006).

12. Schmitt, F. et al. Transient electronic structure and melting of a charge density wave in $\mathrm{TbTe}_{3}$. Science 321, 16491652 (2008).

13. Tomeljak, A. etal. Dynamics of photoinduced charge density wave to metal phase transition in $\mathrm{K}_{0.3} \mathrm{MoO}_{3}$. Phys. Rev. Lett. 102, 066404 (2009).

14. Schäfer, H. et al. Disentanglement of the electronic and lattice parts of the order parameter in a $1 D$ charge density wave system probed by femtosecond spectroscopy. Phys. Rev. Lett. 105, 066402 (2010)

15. Miller, R. J.D. etal. 'Making the molecular movie': first frames. Acta Crystallogr. A66, 137156 (2010).

16. Williams, P.M., Parry, G. S. \& Scruby, C. B. Diffraction evidence for Kohn anomaly in 1T TaS 2 . Phil. Mag. 29, 695699 (1974).

17. Wilson,J.A. Di Salvo. F.J.\& Mahajan, S. Charge density waves and superlattices in the metallic layered transition metal dichalcogenides. Adv. Phys. 24, 117201 (1975).

18. Clerc, F. et al. Lattice distortion enhanced electron phonon coupling and Fermi surface nesting in 1T TaS 2 . Phys. Rev. B 74, 155114 (2006),

19. Sipos, B. et al. From Mott state to superconductivity in $1 \mathrm{~T} \mathrm{TaS}$. Nature Mater. 7 , 960965 (2008).

20. Fazekas, P. \& Tosatti, E. Electrical, structural and magnetic properties of pure and doped IT TaS 2 . Phil. Mag. B 39, 229244 (1979).

21. Scruby, C. B., Williams, P. M. \& Parry, G. S. The role of charge density waves in structural transformations of $1 \mathrm{~T} \mathrm{TaS}$. Phil. Mag. 31, 255274 (1975).

22. Als Nielsen, J. \& McMorrow, D. Elements of Modern X ray Physics Ch. 4.4.5 (Wiley, 2001).

23. Duffey, J. R., Kirby, R. D. \& Coleman, R. V. Raman scattering from IT TaS 2 . Solid State Commun. 20, 617621 (1976).

24. Siwick, B. J., Dwyer, J. R., Jordan, R. E. \& Miller, R. J. D. An atomic level view of melting using fermtosecond electron diffraction. Science 302, 13821385 (2003).

25. Chergui, M. \& Zewail, A. H. Electron and $X$ ray methods of ultrafast structural dynarnics: advances and applications. ChemPhysChem 10, 2843 (2009)

26. Sciaini, G. et al. Electronic acceleration of atomic motions and disordering in bismuth. Nature 458, 5659 (2009)

27. Sokolowski Tinten, $K$. et al. Femtosecond $X$ ray measurement of coherent lattice vibrations near the Lindemann stability limit. Nature 422, 287289 (2003).

28. Johnson, S. L. et al. Directly observing squeezed phonon states with femtosecond $X$ ray diffraction. Phys. Rev. Lett. 102, 175503 (2009)

29. Fritz, D. M. etal. Ultrafast bond softening in bismuth: mapping a solid's interatomic potential with $X$ rays. Science 315,633636 (2007)

30. Beaud, P. et al. Ultrafast structural phase transition driven by photoinduced melting of charge and orbital order. Phys. Rev. Lett. 103, 155702 (2009).

Supplementary Information is linked to the online version of the paper at www.nature.com/nature.

Acknowledgements We would like to acknowledge discussions with V. V. Kabanov T. Dekorsy, D. Mihailovic, U. Bovensiepen and M. Wolf, and thank A. Nagy for help in preparing the video in Supplementary Material. This research was supported by the Sofja Kovalevskaja Award of the Alexander von Humboldt Foundation, the Center for Applied Photonics and Zukunftskolleg at the University of Konstanz, the Natural Science and Engineering Research Council of Canada and the Canada Foundation for Innovation. M.E. acknowledges financial support through the Stiftung der Deutschen Wirtschaft. H.B. acknowledges financial support from the Swiss NSF and the NCCR MaNEP.

Author Contributions R.J.D.M, and J.D. directed this work. H.B. grew $1 T$ TaS, singl crystals. M.E. and M.K. prepared thin films and performed transmission electron microscopy characterization. G.S., G.M., M.E. and H.S. performed the FED experiments at the University of Toronto. M.B., M.E. and H.S. performed the optical pump probe experiments at the University of Konstanz. M.E., H.S. and J.D. performed the data analysis. J.D., G.S. and R.J.D.M. wrote the paper. All authors contributed to discussions,

Author Information Reprints and permissions information is available at www.nature.com/reprints. The authors declare no competing financial interests. Readers are welcome to comment on the online version of this article at

www.nature.com/nature. Correspondence and requests for materials should be addressed to R.J.D.M. (dimiller@lphys.chem.utoronto.ca) or J.D.

(jure.demsar@uni konstanz.de). 